\title{
Frequency of Formication Symptoms in Injured Motorists and in Normal Controls
}

\author{
Zack Z. Cernovsky, Varadaraj R. Velamoor, Stephan C. Mann, L. Kola Oyewumi, \\ James D. Mendonça, and Larry C. Litman
}

\section{ABSTRACT}

Background: Formication is the sensation or feeling as if insects were crawling on or under the skin. It is observed in a variety of clinical situations including drug intoxications, multiple sclerosis, and diabetic neuropathy, among many others. Furthermore, it can be associated with injuries incurred during motor vehicle accidents (MVAs). We examined the frequency of reports of formication in a normal control sample as well as in a sample of motorists who sustained concussive and whiplash injuries following high impact MVAs. We also evaluated the correlations of the formication to measures of pain, insomnia, and of various post-accident neuropsychological symptoms.

Method: De-identified data on 23 injured motorists (mean age $=38.0$ years, $\mathrm{SD}=12.8$ ) and on 20 normal controls (mean age 42.8 years, $\mathrm{SD}=19.9$ ) were available. All motorists responded to the following True/False item: "I have pain in my body which seems to feel like bugs crawling under the surface of my skin." Their data were also available on the Brief Pain Inventory, PostMVA Neurological Symptoms (PMNS) scale, Insomnia Severity Index, and on the Rivermead Post-Concussion Symptoms Questionnaire. The data of normal controls included responses to the following specific item of the formication questionnaire: "Do you sometimes have an annoying feeling in some of your limbs or in some other part of your body as if insects were crawling on or under your skin?" The participants were to circle one of the following responses: "never, very rarely, sometimes, often, or almost constantly."

Results and Discussion: Almost a third $(30.4 \%)$ of the motorists who sustained whiplash trauma in their MVA reported the formication. In contrast, only one of the 20 normal controls (i.e., 5\%) reported formication (this was an elderly man with MRI documented pathology in lumbosacral spine). Notably, formication correlated significantly with the ratings of "reduced feeling in the limbs" $(r=.55, p=.010)$, but not with ratings of "tingling in the limbs" $(r=.21, p>.05)$.

Conclusion: The painful form of formication has been reported by almost a third of motorists who sustained whiplash injuries in their accident.

Submitted : March 4, 2021

Published : March 26, 2021

ISSN: $2593-8339$

DOI: $10.24018 /$ ejmed.2021.3.2.751

\section{Z. Z. Cernovsky*}

Dept. of Psychiatry, Western University, London, Ontario, Canada. (e-mail: zcernovs ${ }^{\circledR}$ uwo.ca)

V. R. Velamoor

Dept. of Psychiatry, Laurentian and Lakehead Universities, and Western University, Ontario, Canada.

(e-mail: velamoorraj@gmail.com)

S. C. Mann*

Central Montgomery Behavioral Heath, Norristown, PA, USA.

(e-mail: smann1234@aol.com)

L. K. Oyewumi

Departments of Psychiatry, Biomedical and Molecular Sciences, and Psychology, Queen's University, Ontario, Canada.

(e-mail: oyewumik@ queensu.ca)

J. D. Mendonça

Dept. of Psychiatry, Western University, London, Ontario, Canada. (e-mail: Jim_Mendonca@hotmail.com) L. C. Litman

Dept. of Psychiatry, Western University, London, Ontario, Canada. (e-mail: corvette033@hotmail.com)

*Corresponding Authors

Keywords: formication, tingling, numbness, whiplash injury, motorists, normal controls.

\section{INTRODUCTION}

Formication is a type of paresthesia for which a physical cause has not been identified. It is an abnormal sensation resembling that of insects crawling on the skin, in the skin, or under the skin. In many cases, the sensation is associated with pain. However, some patients report no pain or discomfort, but experience disgust or worry that, if there were indeed a potentially harmful insect crawling in the dark (or under the clothing) on the skin, it may be erroneously dismissed by others as unreal, as a "phantom misperception."

Certain patients with the post-concussion and whiplash experience formication: the sensation might be attributed to a neurological injury to certain afferent nerve fibres as a part of whiplash trauma to the spine. The formication is also known to occur in patients with peripheral neuropathy such as in diabetes. Beran's [1] study of peripheral neuropathy mentions the "feeling as if ants are crawling over/under the skin." Formication is reported by some patients with multiple sclerosis, or by persons exposed to certain environmental toxins [2] and can sometimes associated with substance abuse or withdrawal symptoms in addicts, including alcoholics.

Formication is a specific subtype of a more general group of aberrant skin sensations known as paresthesias. In its milder forms, formication may resemble another subtype of paresthesia known as tingling. In their extreme form as medical symptoms, however, these two paresthesias are remarkably different. The subjective experience of tingling involves sensation of an electrical current or electrical discharge running through the limb or another area of the 
body and these sensations can become alarmingly painful. Some patients report sensations similar to bubbles moving rapidly through the limb, almost as bubbles released under pressure from carbonated water. In the extreme, some patients describe the "electrical discharge" as at times causing an excruciating twisting or pulling pain on the tendons or muscles, or a burning, itching, or grinding sensations on the living tissues. However, tingling is neither always extreme nor always associated with pain.

In our study on a group of 141 motorists recovering from high impact car accidents, tingling in the limbs or in other part of the body was reported by $80.9 \%$ of the patients [3] Somewhat related subjective neuropsychological symptoms frequently reported by these 141 motorists were numbness in the limbs or in other part of the body (reported by $76.6 \%$ of the motorists) and reduced feeling in the limbs or in another part of the body (reported by $41.8 \%$ of the motorists) [3].

In its extreme form, formication (sensation of insects crawling over or under the skin) differs extensively enough from symptoms such as tingling, numbness or reduced feeling. It deserves special attention as a neurological and neuropsychological phenomenon. Although some patients with formication indeed also experience stabbing, piercing, prickling, or tingling pain, it is advisable to restrict the definition of formication to those with sensations of insects moving on or under the skin, thus avoiding falsely diagnosing formication in patients experiencing only "pins and needles," or "tingling."

The formication is at times somewhat incorrectly conceptualized as involving tactile hallucinations. One of the phenomenological and diagnostic differences between the hallucination of insects and the formication can be the extent to which the patient assumes the insect infestation is real. In general, patients correctly diagnosed with the formication rather than with a hallucination of insects are those who are able to visually check and thus satisfactorily rule out the presence of real insects on their body.

In contrast, the tactile hallucinations of insects moving over the skin can be associated with other psychotic symptoms as a part of major mental illness. However, in many cases, there is no rigid dividing line between hallucination and formication. For example, psychostimulant drugs such as methylphenidate [4] or methamphetamine, cocaine [5] or withdrawal from alcohol in a chronic alcoholic can initially produce the formication-like tactile sensations of insects crawling over the skin that might escalate to tactile and visual hallucinations in some patients. Then, the patients might report even bugs crawling over the surroundings.

Unlike in the formication, pre-existing intrapsychic personality factors seem important in generating certain types of hallucinations. Thus, a person with a phobia of spiders who ingested a hallucinogenic substance might experience frightening visual and tactile hallucinations of spiders crawling over the body without any such specific pre-existing neurophysiologically determined tactile paresthesia.

Patients with the fixed belief that the presence of insects is real may act accordingly, e.g., scratching the skin, using potentially toxic desinfectants, or even cutting into their skin to detect hidden insects. Such patients meet the diagnosis of delusional parasitosis [4] (delusion of being invaded by insects), also known as delusional infestation [6]. The review by Freudenmann and Lepping [7] reported that the mean number of cases of delusional infestation "per institution or hospital and year has been found to range from 0.6 ... to 20" and that "Well over 1,400 definite cases have been published in the literature ..." The usual treatment is via antipsychotic medications such as olanzapine.

In contrast, the formication usually requires no need for psychiatric intervention. For example, the formication that occurs in injured motorists seems clearly enough related to the jolting trauma of whiplash from their accident.

The present study evaluates the frequency of reports of formication in a normal control sample and in a sample of motorists who sustained concussive and whiplash injuries in high impact motor vehicle accidents (MVAs). We also examine the correlations of the formication to measures of pain, insomnia, and of various subjective post-accident neuropsychological symptoms such as the tingling, numbness, and reduced feeling in the limbs, or reduced muscular control over the limbs.

\section{STUDY I: METHOD}

The Study I examined the frequency of reports of the formication in a sample of patients recovering from high impact MVAs. The data were available on their responses to the Item 39 of the Structured Inventory of Malingered Symptomatology (SIMS).[8] SIMS Item 39 is as follows: "I have pain in my body which seems to feel like bugs crawling under the surface of my skin." It appears that the authors of the SIMS (Glenn Smith and Gary Burger) were unaware that formication is experienced by patients with certain type of spinal injuries, neurologic disease such as multiple sclerosis, or after accidental exposure to some toxic chemicals because they fallaciously considered reports of this symptom as indicative of malingering. Their SIMS can no longer be considered a legitimate psychological test due to its methodological flaws [9]-[13]. However, the known responses of injured motorists to its Item 39 could provide us with an estimate of frequency of the formication in that clinical group.

The responses to Item 39 were available as a part of deidentified archival data from 23 survivors of high impact motor vehicle accidents (MVAs). The group consisted of 8 males and 15 females, age 19 to 60 years (mean age $=38.0$, $\mathrm{SD}=12.8$ ), with education from 10 to 18 years (mean=14.1 years, $\mathrm{SD}=1.9$ ). The time elapsed since the patient's MVA ranged from 7 to 217 weeks, with the average at 81.5 weeks $(\mathrm{SD}=55.8)$. They all still experienced active post-accident symptoms that required therapy. Their average scores were $17.2(\mathrm{SD}=11.0)$ on the Post-MVA Neurological Symptoms scale [14], $6.3(\mathrm{SD}=1.3)$ on the "average pain" item of the Brief Pain Inventory [15], and 23.7 ( $\mathrm{SD}=3.0)$ on Morin's Insomnia Severity Index [16]. All patients in this sample could be classified as experiencing some degree of the postconcussion syndrome (scores ranging from 24 to 58 on the Rivermead scale [17], [18], with mean=37.4, $\mathrm{SD}=13.2$ ).

In all 23 cases, the collision damaged the car to the extent of being deemed by insurance assessors as not worthy of repair. Such high impact accidents are too severe to not involve injuries of neuropsychological nature, especially symptoms in the post-concussion whiplash spectrum. 


\section{STUDY I: RESUlTS}

Almost a third $(30.4 \%)$ of the injured motorists reported the painful type of the formication with the sensations of insects crawling under the surface of the skin, as formulated by the Item 39 "I have pain in my body which seems to feel like bugs crawling under the surface of my skin." One of the possible neurologic explanations of such sensations is the injury to some afferent nerve fibers during the cervical, lumbosacral, or thoracic whiplash (WAD-C, WAD-LS, or WAD-T) [19] sustained in the MVA.

The True/False responses to this Item 39 were not significantly correlated ( $p>.05,2$-tailed) to the patients' pain ratings on the Brief Pain Inventory, or to their scores on the Insomnia Severity Index, or scores on the Rivermead PostConcussion Symptoms Questionnaire, or on the Post-MVA Neurological Symptoms (PMNS) scale. The only exception was the correlation of $\mathrm{r}=.55$ of formication to reports of "reduced feeling in the limbs" (Item 7 of the PMNS scale, or see also in its updated version, the Subjective Neuropsychological Symptoms Scale [3]).

The presence of formication was uncorrelated to age $(\mathrm{r}=.02)$ but correlated significantly with gender $(\mathrm{r}=.51)$. As mentioned, the sample of injured motorists consisted of 8 males and 15 females. Five of the 8 males (i.e., 62.5\%), but only $2(13.3 \%)$ of the 15 females reported the formication. The number of positive cases within each gender category is evidently too small for generalizations: data from larger samples are needed.

\section{STUdy II: METHOD}

The Study II examined de-identified archival data available on the formication in a community sample of 20 normal controls. The group consisted of 11 males, 9 females, mean age 42.8 years, $S D=19.9$, age range 23 to 79 years.

All 20 persons completed a questionnaire about their subjective experiences with tingling, numbness, or formication in the limbs or in other parts of their body.

The full text of the questionnaire is reproduced in Table I.

\section{STUdy II: RESUltS}

Only one of the 20 normal controls, a 74 year old male, reported that he "sometimes" experiences formication on the surface of his feet and legs, but without any pain or discomfort. This man has a history of bulging discs in lumbosacral spine, documented via recent MRIs. He also reported feeling "sometimes" tingling and "very rarely" numbness.

Three of the 20 normal controls reported an annoying tingling (one "very rarely," and two "sometimes").

Four normal controls reported an annoying numbness (2 "very rarely" and another two "sometimes").

All those who reported tingling also reported numbness: thus, only 4 of the 20 normal controls experienced these symptoms. One of these four was the man who reported formication.

It is noteworthy that all these persons who reported tingling, numbness, or formication were at least 55 year old (ages 55, 67, 70, and 74 years).

TABLE I: FORMICATION QUESTIONNAIRE

INSTRUCTION: An accident or medical illness can disrupt normal functioning of nerve fibers that conduct information from various parts of the body to the human brain, as follows. Item:

Please circle your

1. Do you sometimes feel an annoying tingling in some of your limbs or in some other part of your body?

response:

never

very rarely

sometimes

often

almost constantly

2. Do you sometimes feel an annoying numbness in some of your limbs or in some other part of your body?

never

very rarely

sometimes

often

almost constantly

3. Do you sometimes have an annoying feeling in some of your limbs or in some other part of your body as if insects never very rarely sometimes were crawling on or under your skin? often

almost constantly

If you answered "Never" to the preceding question, please skip the rest of this questionnaire

4. If you sometimes have the feeling in on the surface of the skin some of your limbs or in some other part under the skin of your body as if insects were crawling or both on or under your skin, please circle if this seems to be:

5. Please also circle if it is causing:

no discomfort, no pain minor discomfort mild pain moderate pain severe pain

6. Please circle all

locations where you feel as if insects were crawling or moving on your skin: 7. Please circle all locations where you feel as if insects were crawling or moving under your skin:

\section{DISCUSSION}

Almost a third $(30.4 \%)$ of the motorists who sustained whiplash trauma in their MVA reported the formication. In contrast, only one person (i.e., 5\%) of a sample of normal controls reported formication: it is noteworthy that this was an elderly man with MRI documented pathology in lumbosacral spine.

There is a paucity of similar statistical studies on the frequency and medical signs of formication. The sensation of insects crawling on or under the skin is often discussed informally in internet self-help groups of patients with multiple sclerosis or among patients with diabetic peripheral neuropathy, but the presence or absence of the formication is rarely assessed formally in interviews in ways that could help generating useful files of statistical data.

Some patients may be reluctant to mention their formication symptoms to a medical professional as they assume that it could be misinterpreted as a sign of psychotic illness.

This is especially relevant in adversarial situations such as when facing a car insurance company-contracted psychologist. The patient anticipates that the psychologist will attempt to discredit any claim for insurance benefits, by undermining the credibility of his self-reports, even to the extent of diagnosing the patient as "psychotic," rather than as suffering from post-concussive and whiplash symptoms. 
Even in non-adversarial situations, the patient may worry that admitting to sensations of insects crawling on or under the skin would detract from his or her being perceived as able to live independently and make independent responsible decisions such as those of financial nature.

As mentioned in the introductory part of this article, very mild forms of formication may be similar to tingling. However, in their extreme form as medical symptoms, formication and tingling are substantially different. This is confirmed by the lack of high and statistically significant correlations observed in our study between formication (as represented by the Item 39) and tingling (Item 5 of the old PMNS scale, see also of its updated version as the SNPSS [3]): the correlation was only $\mathrm{r}=.21, \mathrm{p}=.353,2$-tailed.

The formication correlated significantly $(r=.55, p=.010,2$ tailed) with ratings of "reduced feeling in the limbs" (Item 7 of the PMNS scale, see also the SNPSS). Perhaps, formication could be considered as impairing the normal expected sensory feedback from the limbs by replacing it with an abnormal one.

The limitation of comparing our two studies lies mainly in the different way in which the presence of formication was determined in the sample of injured motorists as opposed to the method used with normal controls. Above all, the True/False question to motorists specified both the presence of pain and of insects crawling under the skin, as follows: "I have pain in my body which seems to feel like bugs crawling under the surface of my skin."

In the question posed to our sample of normal controls, the formication was described as an annoying feeling, but the presence of pain was not required: "Do you sometimes have an annoying feeling in some of your limbs or in some other part of your body as if insects were crawling on or under your skin?" It is also noteworthy that the formication as evaluated in the normal control sample included only the sensation of insects on the skin (not necessarily under the skin).

\section{CONCLUSIONS}

Almost a third (30.4\%) of the motorists who sustained whiplash trauma in their MVA reported the formication. In contrast, only one person (i.e., 5\%) of a sample of normal controls reported formication: an elderly man with MRI documented pathology in lumbosacral spine. At present, very little is known about statistical frequencies of the formication and its particular subjective forms in various clinical groups, i.e., phenomenological differences such as in presence versus absence of pain, or in the sensation of insects crawling on versus under the skin. Research data should help to clarify neurologic and neuropsychological correlates of the subjective forms of formication.

\section{REFERENCES}

[1] Beran R. Paraesthesia and peripheral neuropathy. Australian Family Physician. 2015; 44(3):92-5.

https://www.racgp.org.au/download/Documents/AFP/2015/March/AF P-2015-3-Beran.pdf.

[2] Thawani S, Wang B, Shao Y, Reibman J and Marmor M. Time to Onset of Paresthesia Among Community Members Exposed to the World Trade Center Disaster. International Journal of Environmental Research and Public Health. 2019, 16, 1429.

[3] Cernovsky ZZ, Litman LC, Mann SC, Oyewumi LK, Bureau Y, Mendonça JD, Diamond DM, and Raheb H. Validation of the
Subjective Neuropsychological Symptoms Scale (SNPSS) in Injured Motorists. Archives of Psychiatry and Behavioral Sciences. 2021;4(1):6-13.

[4] Wykoff RF. Delusions of parasitosis: a review. Reviews of Infectious Diseases. 1987;9(3):433-7.

[5] Mitchell J, Vierkant AD. Delusions and Hallucinations of Cocaine Abusers and Paranoid Schizophrenics: A Comparative Study. Journal of Psychology. 1991;125(30:301-310.

[6] Reich A, Kwiatkowska D, Pacan P. Delusions of Parasitosis: An Update. Dermatology and Therapy (Heidelberg).2019;9:631-638. https://doi.org/10.1007/s13555-019-00324-3.

[7] Freudenmann RW and Lepping P. Delusional Infestation. Clinical Microbiology Reviews. 2009; 22(4):690-732.

[8] Smith GP, Burger GK. Detection of malingering: Validation of the Structured Inventory of Malingered Symptomatology (SIMS). Journal of the American Academy on Psychiatry and Law. 1997; 25:180-183.

[9] Cernovsky ZZ, Mendonça JD, Ferrari JR, Sidhu G, Velamoor V, Mann $\mathrm{SC}$, et al. Content Validity of the Affective Disorder Subscale of the SIMS. Archives of Psychiatry and Behavioral Sciences. 2019; 2(2):3339.

[10] Cernovsky Z, Bureau Y, Mendonça J, Velamoor V, Mann S, et al Validity of the SIMS Scales of Neurologic Impairment and Amnestic Disorder. International Journal of Psychiatry Sciences. 2019;1(1):1319.

[11] Cernovsky ZZ, Mendonça JD, Ferrari JR, Bureau YRJ. Content validity of SIMS low intelligence scale. International Journal of Research in Medical Science. 2019; 1(1):21-25.

[12] Cernovsky Z, Mendonça JD, Oyewumi LK, Ferrari JR, Sidhu G, Campbell R, et al. Content Validity of the Psychosis Subscale of the Structured Inventory of Malingered Symptomatology (SIMS). International Journal of Psychology and Cognitive Science. 2019;5(3):121-127.

[13] Cernovsky ZZ, Mendonça JD, Ferrari JR. Meta-Analysis of SIMS Scores of Survivors of Car Accidents and of Instructed Malingerers. Archives of Psychiatry and Behavioral Sciences. 2020;3(1):1-11.

[14] Cernovsky ZZ, Istasy PVF, Hernández-Aguilar ME, Mateos-Moreno A, Bureau Y, Chiu S. Quantifying Post Accident Neurological Symptoms Other than Concussion. Archives of Psychiatry and Behavioral Sciences. 2019, Volume 2, Issue 1, 5054.

[15] Cleeland CS. The Brief Pain Inventory - User Guide. Houston, TX: The University of Texas - M. D. Anderson Cancer Center, 2009.

[16] Morin CM, Belleville G, Bélanger L, and Ivers H. The insomnia severity index: psychometric indicators to detect insomnia cases and evaluate treatment response. Sleep. 2011; 34:601-608.

[17] King NS, Crawford S, Wenden FJ, Moss NEG, Wade DT. The Rivermead Post Concussion Symptoms Questionnaire: a measure of symptoms commonly experienced after head injury and its reliability. Journal of Neurology. 1995;242:587-592.

[18] Cernovsky ZZ, Mann SC, Velamoor V, Oyewumi LK, Diamond DM, Litman LC. Validation of the Rivermead Post-Concussion Symptoms Questionnaire (RPQ) on Patients Injured in High Impact Car Accidents. Archives of Psychiatry and Behavioral Sciences. 2021;4(1):14-22. doi.org/10.22259/2638-5201.0401003.

[19] Cernovsky ZZ, Mann SC, Velamoor VR, and Oyewumi LK. The Need for Three Separate Parallel WAD Ratings of Whiplash Injuries to Cervical, Lumbosacral, and Thoracic Spine in Clinical Assessments of Injured Motorists. European Journal of Medical and Health Sciences. 2021;3(1):1-6. doi: 10.24018/ejmed.2021.3.1.699.

Zack Z. Cernovsky was born in 1947 and holds the Ph.D. in clinical psychology from the University of Zürich, Switzerland, 1986. He is the professor of psychiatry in the medical school of Western University, Canada Professor Cernovsky has published close to 200 scientific articles in the field of psychiatry and medical psychology, and also chapters in university textbooks.

Varadaraj R. Velamoor graduated in Medicine from Osmania University in India and received his training in Psychiatry in the United Kingdom. He is currently Professor of Psychiatry at the Northern Ontario School of Medicine as well as Professor Emeritus at the Western University School of Medicine. He was previously on Faculty at Cornell University. Professor Velamoor has published over a hundred scientific articles as well as book chapters and psychiatric monographs in the areas of suicidal behaviour, violence, stress at the work place, collaborative care, geriatric psychopharmacology and the Neuroleptic Malignant Syndrome (NMS). His work in NMS is internationally recognized and frequently cited. He has received Fellowships from the Royal College of Psychiatrists, UK, as well as the American Psychiatric Association. 
Stephan C. Mann was born on May 6, 1948 in Philadelphia, Pennsylvania, USA. He received his medical degree from the Sidney Kimmel Medical College of Thomas Jefferson University where he also completed his residency in psychiatry. He worked for the United States Veterans Health Administration for over thirty years and retired as Chief of Mental Health \& Behavioral Sciences at the Louisville VA Medical Center, Louisville, KY. He is currently in private practice. Dr. Mann has previously held faculty positions as Professor of Psychiatry at both the University of Pennsylvania School of Medicine and the University of Louisville School of Medicine. He is a Distinguished Life Fellow of the American Psychiatric Association and is certified by the American Board of Psychiatry and Neurology. He has published almost one hundred scientific publications including three books. Dr. Mann's main research focus includes neuroleptic malignant syndrome, malignant catatonia, tardive dyskinesia, and the neurobiology of schizophrenia

L. Kola Oyewumi completed his medical training from the University of Ibadan, Nigeria, and the residency training in Psychiatry from the University of Ottawa, Canada. He is a Fellow of the Royal College of Physicians and Surgeons of Canada, a Diplomate of the American Board of Psychiatry and Neurology, and A Distinguished Fellow of the Canadian Psychiatric Association. He was a full-time tenured Professor of Psychiatry, Biomedical and Molecular Sciences and Psychology at Queen's University, Kingston, Canada until retirement in 2012. He was then awarded the Professor Emeritus status at Queen's University. Professor Oyewumi has authored numerous peer-reviewed articles, scientific abstracts, presentations, reports, book chapters and the book: Managing Side Effects of Psychotropic Medications - A handbook for healthcare professionals. His research focus was on "Judicious use of psychotropic medications," with emphasis on early detection and intervention in Mental Health; as well as strategies for treatment- resistant Psychoses.

James D. Mendonça obtained his doctorate in psychology at Western University, Canada. Dr. Mendonça is currently an Adjunct Professor in the Department of Psychiatry, Schulich school of Medicine \& Dentistry, Western University, London, Ontario, Canada. Professor Mendonça has published 57 papers and presentations. His research studies dealt with topics such as Suicidal phenomena related to motives, hopelessness, active and passive ideation, thinking dysfunctions; Depression following cardiac surgery; Mood disorders in the bipolar spectrum and patient cognitive schemas regarding treatment. He is currently Editor of "Research Insights," a journal of Parkwood Mental Health, St Joseph's Health Care, London, Canada.

Larry Craig Litman obtained his Ph.D. in clinical psychology from York University, Ontario, Canada. He has taught classes in statistics at that university. Dr. Litman is currently on faculty staff of the Department of Psychiatry, Schulich school of Medicine \& Dentistry, Western University, London, Ontario, Canada. He has published numerous empirical studies in clinical psychology and psychiatry. 